\title{
ÜBER FINNLANDS REZENTE UND SUBFOSSILE DIATOMEEN VI
}

\author{
Karl Mölder und Risto Tynni
}

\begin{abstract}
Mölder, KarL und Tynni, Risto 1972: Über Finnlands rezente und subfossile Diatomeen VI. Bull. Geol. Soc. Finland 44, 141-149.

The article deals with the distribution and ecology of the Cocconeis and Acbnantbes species known in Finland.

Karl Mölder, Kelohongantie 2 C 21, 02120 Tapiola 2 Finnland.

Risto Tynni, Geologische Forschungsanstalt in Finland, 02150 Otaniemi, Finnland.
\end{abstract}

\section{Cocconeis}

Valvarhabitus der Schalen elliptisch, in der einen Schalenhälfte ist eine Raphe, in der anderen eine Pseudoraphe. In der Valvarebene im Randteil der Raphenschale erscheint oft ein gekammerter oder ein hyaliner Ring, zuweilen auch im Randteil der Pseudoraphenschale. Die transapikale Struktur ist meistens auf der Raphen- und der Pseudoraphenhälfte unterschiedlich. Sie besteht allgemein aus Punktierung, die auf der Pseudoraphenhälfte gröber als auf der Raphenhälfte ist. Einige Arten haben transapikale Rippen, Areolierung oder hyaline Längsfurchen.

Die Cocconeis-Arten sind allgemein epiphytisch, sie wachsen an Gefässpflanzen und höheren Algen der Uferzone. Die meisten in Finnland angetroffenen Arten leben in der Uferzone von Süsswasser, Seen und Flüssen, weniger in der seichten Litoralzone der Küste.

Subfossil eine häufige Diatomeengattung. Reichliches Vorkommen erweist eine seichte Sedimentationsphase.

\section{Cocconeis costata Gregory}

Meeresform, die in der Küstenzone zwischen Mittelmeer und Nördlichem Eismeer festgestellt worden ist. Subfossil im interglazialen Tonklumpen von Rouhiala (Brander 1937) und in Tunstajoki (Cleve-Euler).

\section{Cocconeis diminuta Pantocsek}

Synonym: Cocconeis disculus var. diminuta (Pant.) A. Cleve, neg. Hustedt. Beschreibung: siehe Cleve-Euler, 1953.

Halophile Form, die besonders an der Küste des Bottnischen und des Finnischen Meerbusens anzutreffen ist. (Abb. 1).

\section{Cocconeis dirupta Gregory}

Meeresform, die in einer Litorinaablagerung der Gegend von Helsinki festgestellt worden ist (Brander in Brander och Brenner 1933).

Cocconeis disculus (Schumann) Cleve

Synonym: Navicula discula Schumann. Beschreibungen: siehe auch Patrick \& Reimer 1966. 


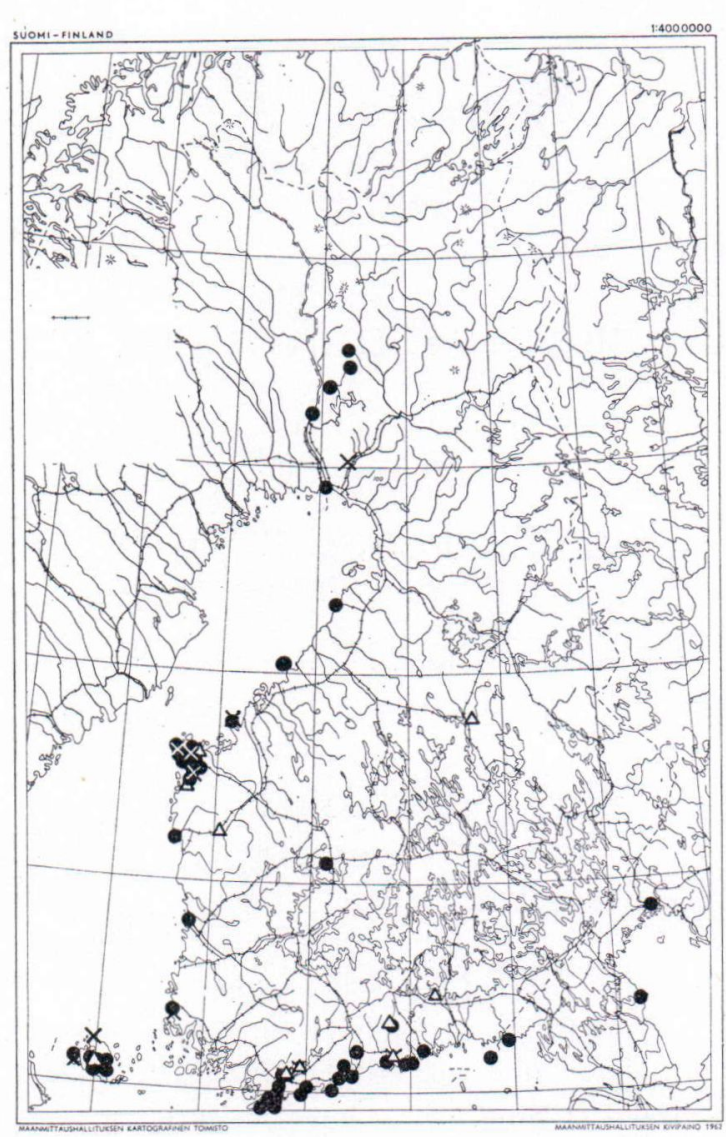

Авв. 1. Rezente Verbreitung von Cocconeis diminuta (Kreuze), C. disculus (Dreiecke) und C. pediculus (Punkte).

Verhältnismässig seltene Süsswasserform. Subfossil vorwiegend in Ancylussee-Sedimenten. (Abb. 1.)

\section{Var. minor Fontell}

Cleve-Euler 1953 hat diese Variation aus einigen finnischen Seen und Sedimentfolgen daigestellt.

\section{Cocconeis pediculus Ehrenberg}

Halophile Form, die in schwach salzhaltigem Küstengebiet am reichlichsten vorkommt. In Binnengewässern wächst die Art vorwiegend in eutrophem, kalkhaltigem Wasser. (Abb. 1.)

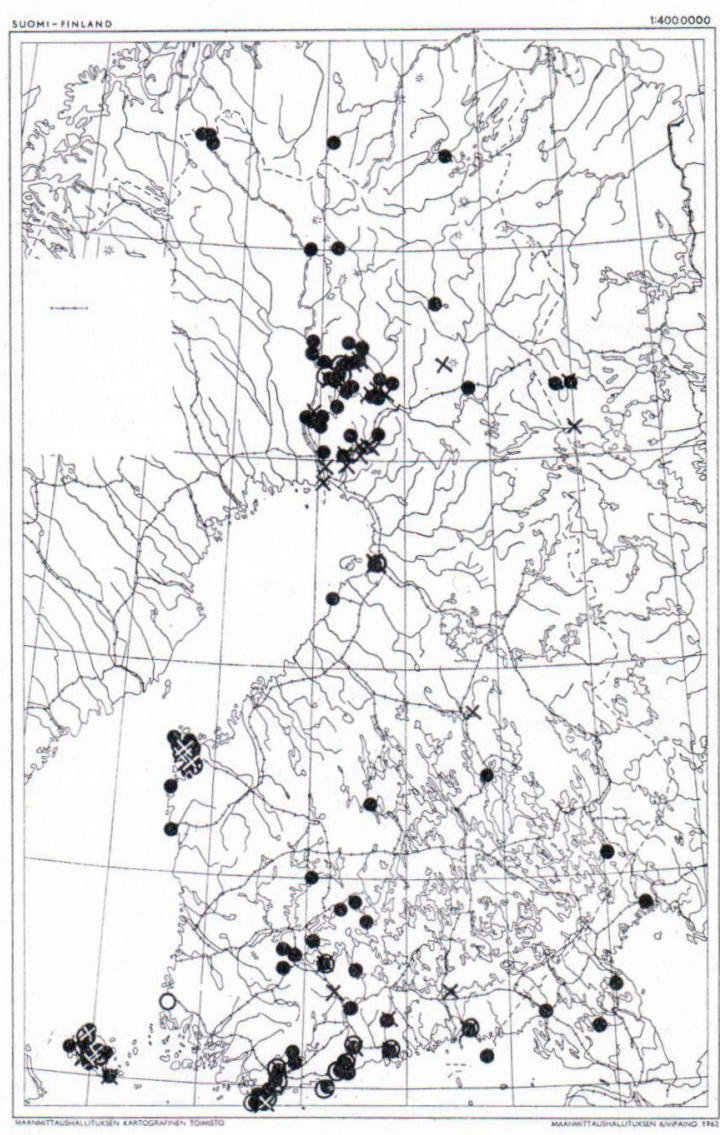

Авв. 2. Rezente Verbreitung von Cocconeis placentula (Punkte), C. p. var. euglypta (Kreuze) und C. p. var. klinoraphis (Ringe).

\section{Cocconeis placentula Ehrenberg}

Die in Finnland häufigste Cocconeis-Art, die in Seen, Flüssen und an der Küste in schwachem Brackwasser wächst (Abb. 2). Alkaliphile Form.

Var. euglypta (Ehr.) Cleve

Synonym: Cocconeis euglypta Ehrenberg.

Neben der Hauptart eine rel. häufige Variation (Abb. 2). Ihr früheres Wachstumsoptimum ist im Februar festgestellt (Mölder \& Tynni 1966) und sie ist eurytherme Kaltwasserform. Im übrigen ist die Ökologie die gleiche wie bei der Hauptart. 


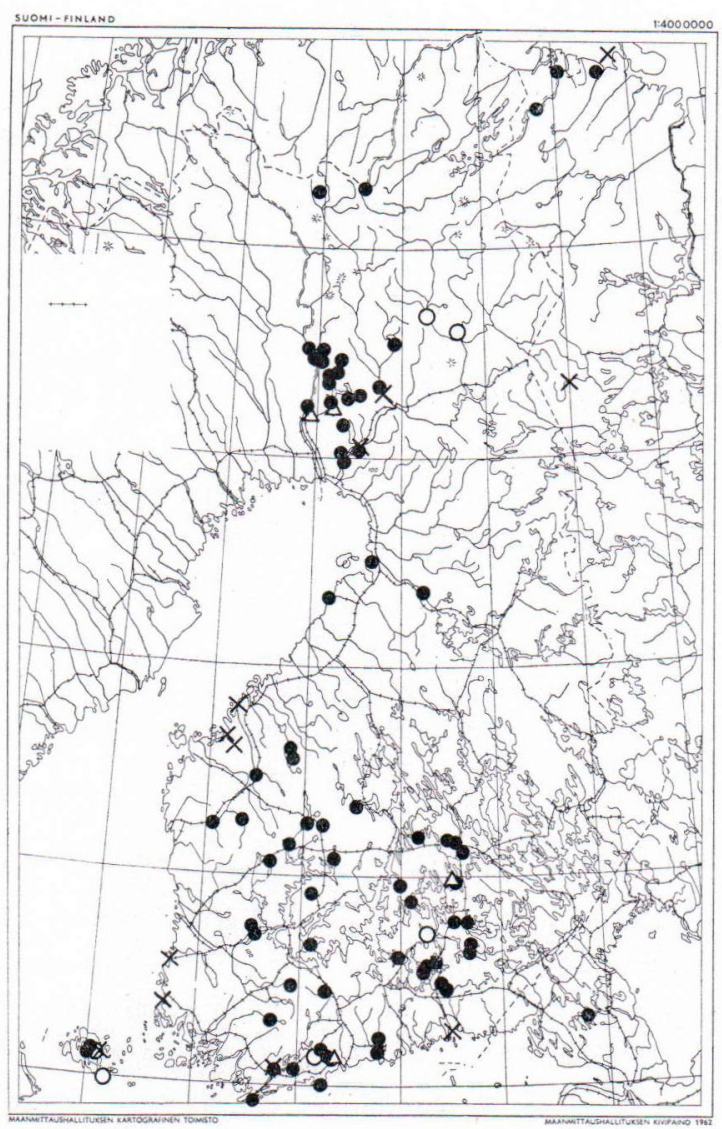

Авв. 3. Rezente Verbreitung von Cocconeis scutellum (Kreuze), Achnanthes affinis (Ringe), A. austriaca var. belvetica (Dreiecke) und $A$. biasolettiana (Ringe).

Var. intermedia (Her. \& Per.) Cleve

Seltene Variation, deren Punktierung gröber ist als bei der Hauptart.

Sie ist subfossil in Satakunta (Aario 1932) und in Ostbottnien (Backman u. Cleve-Euler 1922) festgestellt worden (Cl. E: Cocconeis placentula var. intermedia fo. minor).

\section{Var. klinoraphis Geitler}

Neben der Hauptform eine verhältnismässig allgemein festgestellte Variation (Abb. 2), die sich von jener dadurch unterscheidet, dass Raphe und Pseudoraphe sich in bezug auf die Aplikalachse um bis etwa $5^{\circ}$ gewendet haben.
Var. lineata (Ehr.) Cleve

Synonym: Cocconeis lineata Ehrenberg.

Neben der Hauptart selten angetroffene Variation.

\section{Cocconeis quarnerensis Grunow}

Synonym: Rhaphoneis quarnerensis Grunow.

Meeresform, die u. a. in der südlichen Ostsee eingetroffen worden ist (Hessle \& Wallin 1934).

Subfossil in Litorina-Postlitorina-Sedimenten selten sowie im interglazialen Tonklumpen von Rouhiala (Brander 1937).

\section{Cocconeis scutellum Ehrenberg}

Synonyme: Rhaphoneis scutellum Ehrenberg, Cocconeis scutellum var. maior, var. ornata, var. ampliata, var. baldjukiana Grunow et cet.

Häufige Meeresform sowohl an der gegenwärtigen Küste (Abb. 3) als auch in litoralen Meeressedimenten.

\section{Var. parva Grunow}

Synonym: Cocconeis scutellum var. minuta Grunow.

Häufig neben der Hauptart wachsende kleine Variation.

Var. stauroneiformis W. Smith

Seltene Meeresform, u. a. in der Pojobucht festgestellt (Mölder).

\section{Cocconeis thumensis Mayer}

Beschreibungen: siehe auch Hustedt 1950.

Eine kleine Cocconeis-Art, die in einigen Gewässern Lapplands und in der Pojobucht in seltenem Vorkommen festgestellt worden ist.

Cleve-Euler (1953) hat auch Cocconeis japonica A. Cleve, C. cholnokiani Cleve-Euler und C. thomasiani Brun in Lappland dargestellt. Sie gehören in denselbe Formkreis wie $C$. thumensis und die vielleicht ihr als Synonyme zichen soll.

\section{Achnanthes}

Längliche, oft an den Enden verschmälerte Schalen. Die Schalenhälften mehr oder weniger verschieden, die eine har eine Raphe, die andere 
eine Pseudoraphe, die eine exzentrische Lage einnehmen kann. Oft ist die Transapikalstruktur in den Schalenhälften abweichend. Bei einigen Arten zeigt die Pseudoraphenschale im Randteil des Mittelfeldes eine hufeisenförmige Figur. Bei vielen Arten sind die Schalen in bezug auf die Transapikalachse sanft, flach V-oder U-förmig geknickt, was am deutlichsten in Gürtelbandansicht hervorttitt.

Die Gattung ist verhältnismässig umfangreich, so dass bei der Bestimmung der Arten die Anwendung systematischer Definitionsschemen von Vorteil ist (u. a. Hustedt 1933, Cleve-Euler 1953). In der vorliegenden Untersuchung sind aus Finnland 81 Arten und Variationen dargestellt. Bei der Unterscheidung kleinerer Formen setzt die Maximalvergrösserung des Lichtmikroskpops schon Begrenzungen, weswegen in Zukunft möglicherweise das sich entwickelnde Elektronenmikroskop und seine Ingebrauchnahme auch über Zusammensetzung dieser Diatomeengattung in Finnland weiteren Aufschluss bringen werden.

Die Achnanthes-Arten sind hauptsächlich Aufwuchsformen, nur die marine $A$. taeniata ist pelagische Form. Die meisten Arten sind Süsswasserformen, und ihr Vorkommen hängt mit der Uferzone zusammen. Viele, besonders die kleinsten Arten sind aerophil und wachsen an feuchten Steinen und Moosen, entweder ausschliesslich oder neben der Lebensmöglichkeit in Binnengewässern.

Unter den subfossilien Achnanthes-Formen am wichtigsten sind die in den Sedimenten der Ostseestadien angetroffenen: im Ancylussee $A$. clevei, in marinem Stadium, besonders im Litorina- und im Postlitorinameer $A$. brevipes, $A$. longipes, $A$. bauckiana, $A$. delicatula, $A$. taeniata.

\section{Achnanthes affinis Grunow}

Synonym: Microneis affinis Cleve.

Verhältnismässig seltene Achnanthes-Art, die sowohl in süssem als auch in schwach salzhaltigem Küstenwasser festgestellt worden ist (Abb. 3). Alkaliphile Form.
Achnanthes anceps A. Cleve

Möglicherweise eine subarktische Form, die aus Finnland nur vom Fl. Vaskojoki dargestellt worden ist (Cleve-Euler). Kennzeichen sind: Länge 21--34 $\mu$, an den Enden schnauzenförmig vorgezogene Form sowie sehr dichte Transapikalstreifung $(30 / 10 \mathrm{u})$.

\section{Achnanthes arcuata A. Cleve}

Nordisch-arktische Süsswasserart, die von Cleve-Euler (1953) u. a. vom See Kemijärvi dargestellt worden ist.

\section{Acbnanthes austriaca Hustedt}

Nordisch-alpine Süsswasserart. Krasske (1949) hat sie im Gebiet Kilpisjärvi in Moosen als häufig erkannt. Alkaliphile Form.

\section{Var. belvetica Hustedt}

Ausser in Lappland ist die Variation auch in Mittel- und Südfinnland, u. a. von Meriläinen (1969) in dem meromiktischen See Valkiajärvi, selten fesgestellt worden (Abb. 3). Alkaliphile Form.

\section{Achnanthes biasolettiana (Kütz.) Grunow}

Synonyme: Synedra biasolettiana Kützing, Achnanthes biasolettiana fo. minuta Grunow, $A$. b. var. perminuta V. Heurck.

Die in Finnland anzutreffende Form ist klein (Cleve-Euler: var. minuta), und sie wächst besonders in eutrophen Gewässern, alkaliphil. Auch ist sie an der Küste in schwach salzhaltigem Wasser festgestellt worden. Abb. 3.

Var. aapajärvensis (A. Cleve) n. comb.

Eine $\operatorname{der}$ A. biasolettiana ähnelnde kleine Form, die von Cleve-Euler (1953) aus FinnischLappland als sehr selten dargestellt worden ist.

\section{Var. sublinearis Grunow}

Meriläinen (1969) hat die Variation aus dem meromiktischen See Valkiajärvi dargestellt. 


\section{Achranthes bicapitata Hustedt}

Seltene Art, die von Meriläinen (1969) für den obengenannten Valkiajärvi beschrieben worden ist.

\section{Acbnanthes bottnica Cleve}

Synonym: Achnanthes clevei var. bottnica Cleve.

Seltene Süsswasserart, die auch in schwach salzhaltigem Wasser wächst. Die meisten Beobachtungen sind von Binnengewässern (Abb. 4) und Süsswassersedimenten.

\section{Acbnanthes brevipes Agardt}

Synonym: Acbnantbidium brevipes (Ag.) Cleve.

An der Küste bis zur Höhe von Vaasa eine häufige Brack- und Salzwasserform (Abb. 4). In marinen Sedimenten der Ostseephasen, u. a. im interglazialen Tonklumpen von Rouhiala (Brander 1943) und in der Spätglazialzeit umgelagerten Ton von Somero (Tynni in Donner, Gardemeister 1971).

\section{Var. intermedia (Kütz.) Cleve}

Synonym: Acbnanthes intermedia Kützing.

Kleinere und in schwächer salzhaltigem Wasser lebende Form, die auch im Wasser vor Tornio festgestellt worden ist.

\section{Achnanthes calcar Cleve}

Synonym: Cocconeis calcar A. Cleve.

Verhältnismässig seltene nordisch-alpine Klarseeform. Die meisten Funde in Lappland (Abb. 4). Alkaliphile Form.

Subfossil u. a. in Ancylussee-Sedimenten.

\section{Achnanthes clevei Grunow}

Synonym: Actioneis clevei Cleve.

Form süssen, klaren Wassers, die auch in schwach salzhaltigem Küstenwasser des Bottnischen Meerbusens wächst (oligohalob), desgleichen in fliessendem Wasser. (Abb. 4.) Nach Hustedt Tiefenform. Alkaliphile Form mesound eutropher Seen und Flüsse. Des weiteren

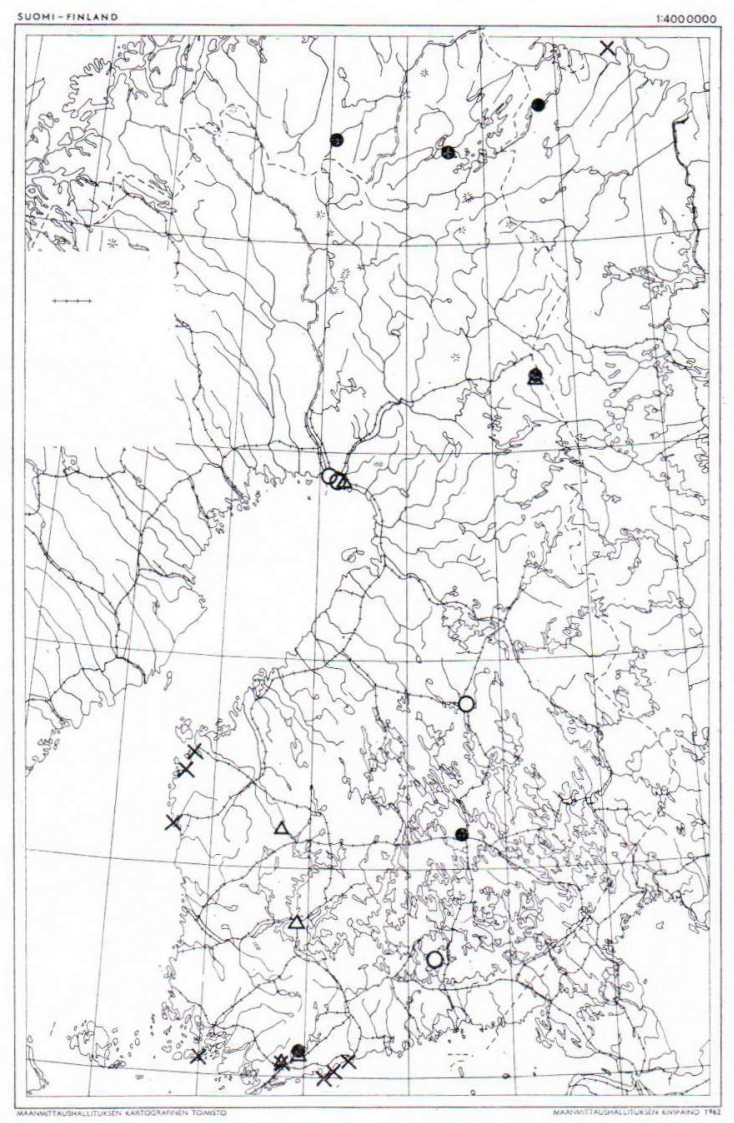

Авв. 4. Rezente Verbreitung von Acbnantbes bottnica (Ringe), A. brevipes (Kreuze), A. calcar (Punkte) und A. clevei (Dreiecke).

in Nordamerika (Florin 1970), Japan (Iwaki 1968), Jugoslawien (Jurilj 1954).

Oft in Sedimenten süssen Wassers (Ancylussee - Kleinsee) in baltischem Gebiet festgestellt.

Var. rostrata A. Cleve, Hustedt

Neben der Hauptart, aber seltener als diese.

Achnanthes coarctata (Breb.) Grunow

Synonyme: Acbnanthidium coarctatum Brébisson, Achnanthes coarctata var. constricta Krasske.

Selten beobachtete Süsswasserform (oligohalob), die im allgemeinen an Steinen und in 


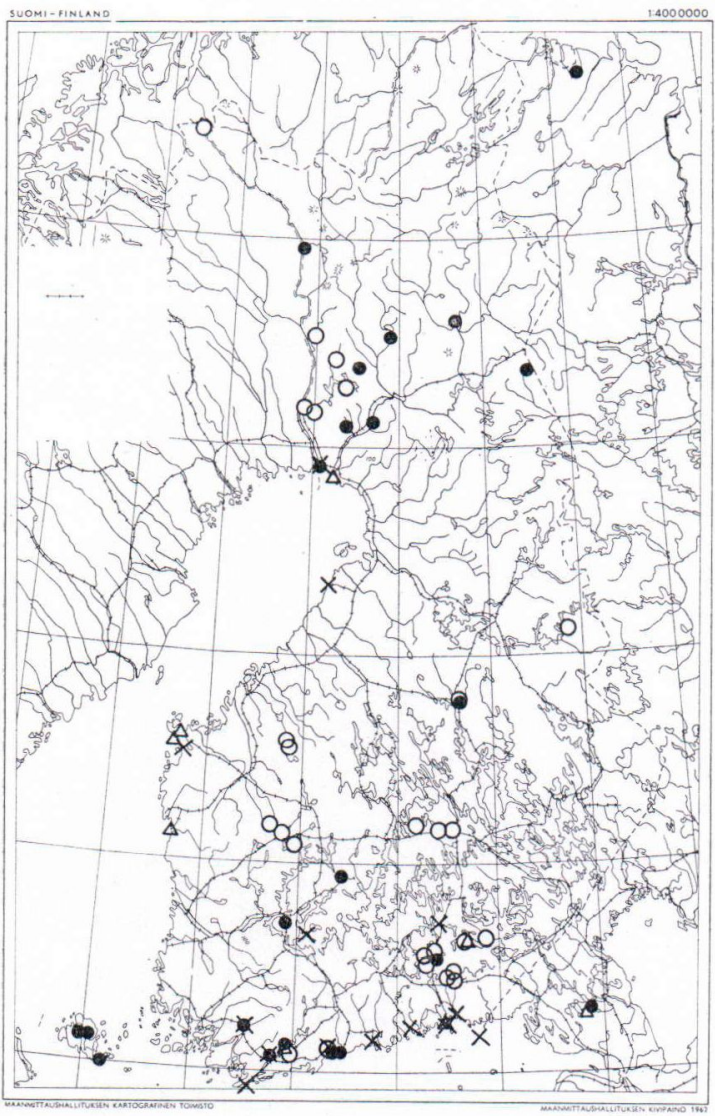

Авв. 5. Rezente Verbreitung von Acbnantbes conspicua (Kreuze), A. delicatula (Dreiecke), A. didyma (Ringe) und A. exigua (Punkte).

Hochwassergebiet in Moos vorkommt; aerophil. Bei der Bestimmung besteht die Gefahr, die Art mit der Brackwasserform $A$. brevipes var. intermedia an. zu verwechseln. Round (1959) hat die Art und die folgende Variation vom Schärenhof Tammisaari her, aus Kleintümpelsedimenten, dargestellt. Nach Cleve-Euler (1953) hat Fontell die Art subfossil in Maaninka (Savo) angetroffen.

\section{Var. elliptica Krasske}

Round (1959) hat die Variation in Lappland in Sedimenten des Flusses Muonionjoki sowie im Schärenhof von Tammisaari in Tümpelsedimenten beobachtet. Variation ähnelt der Brack- wasserform Achnanthes brevipes var. parvula (Kütz.) Cleve.

\section{Achnantbes conspicua A. Mayer}

Nach Hustedt ist die Art alkaliphil, in den Alpen und im Baltikum in Seen wachsend. Cleve-Euler hat sie weder in Schweden noch in Finnland festgestellt. Nach den Beobachtungen Mölders ist die Art im Küstengebiet häufig, desgleichen ausserdem in einigen Binnenseen. (Abb. 5). Somit müsste es sich um eine Form süssen und schwach salzhaltigen Wassers handeln.

Var. brevistriata Hustedt

Seltene Variation. Subfossiı häufiger.

\section{Achnanthes crucicera Østrup}

Kleine, zartstrukturierte Form, die zusammen mit der nahestehenden Var. capitata von CleveEuler (1953) u. a. aus Lappland, vom AngeliVaskojoki, dargestellt worden ist.

\section{Achnanthes delicatula (Kütz.) Grunow}

Synonyme: Achnantbidium delicatulum Kützing, Microneis delicatula Cleve.

Die Struktur unterscheidet sich von der von Hustedt dargestellten in erster Linie dadurch, dass in der Pseudoraphenhälfte die Transapikalstreifen beinahe parallel und nicht radial verlaufen. Brackwasserform (Abb. 5), selten auch in Süsswasser.

\section{Achnanthes depressa (Cleve) Hustedt}

Nordeuropäische Süsswasserform. Einige Fundorte aus Lappland (Krasske, Cleve-Euler, Salminen), Vuokatti (Hustedt) und Südfinnland (Mölder).

\section{Var. lanceolata A. Cleve}

Seltene Variation, von Cleve-Euler aus dem See Nilojärvi in Kuusamo dargestellt. 


\section{Achnanthes didyma Hustedt}

Beschreibungen: vergleich auch Navicula contenta var biscutiformis Cl.-E. nach Hustedt 1967.

Kleine Süsswasserform, in Finnland verhältnismässig häufig, besonders in Lappland (Abb. 5). Nordisch-alpin.

\section{Achnanthes dispar Cleve}

Seltene Form von schwach brackigem sowie von Süsswasser (Hustedt und Cleve-Euler: halophil). Cleve hat die Art für das Gewässer vor Tornio belegt.

In Sedimenten des baltischen Raumes verhältnismässig häufig.

\section{Var. angulata Hustedt}

Subfossil oft neben der Hauptform.

\section{Achnanthes elliptica (Cl.) A. Cleve}

Eine $\operatorname{der} A$. lanceolata var. elliptica wesentlich ähnelnde Form, die von Cleve-Euler aus vielen Seen und Flüssen Finnlands, besonders aus Lappland, dargestellt worden ist. In anderen Bestimmungen ist die Form allgemeiner an $A$. lanceolata var. elliptica angeschlossen worden. Die Diatomeetypenkombination, die den betreffenden Habitus und im Randteil der Pseudoraphe einen hufeisenförmigen Fleck aufweist, in drei Arten aufzuteilen, ist unseres Frachtens angebracht. Dabei liegen die wichtigsten unterscheidenden Merkmale in den Transapikalstreifen der Pseudoraphenschale. A. elliptica: Streifen lang (Raphenund Pseudoraphenarea schmal) und relativ dicht, A. lanceolata var. elliptica: Str. kürzer und lichter, A. östrupi: Str. kurz und rel. dicht.

\section{Achnanthes exigua Grunow}

Synonyme: Stauroneis exilis Kützing, Microneis exigua Cleve.

Süsswasserform, besonders in eutrophen Seen in Süd- und Mittelfinnland (Abb 5). Eigentlich eine Form warmen Wassers (u. a. in Java und Sumatra, Hustedt 1938; in Indien, Gandhi 1964),

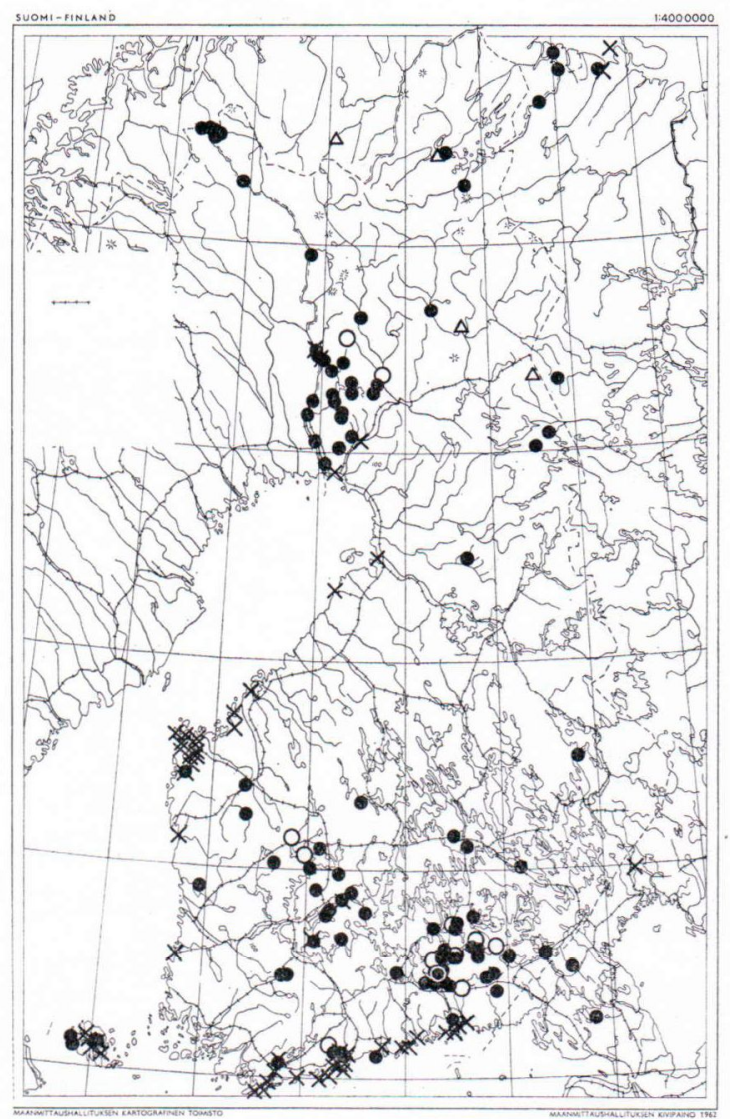

Aвв. 6. Rezente Verbreitung von Acbnanthes exilis (Ringe), A. flexella (Punkte), A. bauckiana (Kreuze) und A. b. var. rostrata (Dreiecke).

aber im weiteren Sinne eurytherm. Nach Hustedt (1957) wächst die Art auch im Laubmoos auf aerischen Standorten, alkaliphil.

Var. constricta Torka

Neben der Hauptform rel. Seltene Variation.

Var. beterovalvata Krasske

Neben der Hauptart oder auch ausschliesslich vorkommende rel. gemeine Variation.

\section{Achnanthes exilis Kützing}

Verhältnismässig seltene Süsswasserform. (Abb. 6.) 
Acbnanthes flexella (Kütz.) Brun

Synonyme: Cymbella flexella Kützing, Achnanthidium flexellum Brèb., Eucocconeis flexella Cleve.

Häufige Art in Seen Süd- und Nordfinnlands. (Abb. 6.)

\section{Var. alpestris Brun}

Synonym: Eucocconeis minuta var. alpestris (Brun) Cleve

Vielfach an denselben Stellen wie die Hauptform, aber eine bedeutend seltenere nördliche, im Gebirge lebende Form.

\section{Acbnanthes fragilarioides Petersen}

Arktische Süsswasserform. Subfossil selten festgestellt.

\section{Achnanthes gracillima Hustedt}

Eigentliche östliche (Japan) Art, die vereinzelt in Lappland, den See Kuolajärvi (Cleve-Euler 1934) und Kilpisjärvi (Krasske 1949), festgestellt worden ist.

\section{Achnanthes bauckiana Grunow}

An den Küsten Finnlands häufigste Vertreterin der Gattung Achnanthes. Ausserdem einzelne Vorkommen in einigen Binnenseen. (Abb. 6.)

\section{Var. rostrata Schulz}

Rezent aus Lappland, subfossil aus ålandischen Litorinasedimenten (Cleve-Euler 1935: A. delicatula var. rostrata) dargestellte seltene Variation (Abb. 6). Auf Grund des Obigen eine Form süssen und schwach brackigen Wassers.

\section{Achnanthes bolsti Cleve}

Eine in Nordeuropa vorkommende Süsswasserform. In Finnland rezent nur in Lappland u. a. in Varpupää an Moosen festgestellt. Möglicherweise eine azidophile Form.

\section{Achnanthes bungarica Grunow}

Synonym: Achnantbidium bungaricum Grunow, Microneis bungarica Cleve.
Eine im Küstengebiet und einigen Seen von Kuusamo selten angetroffene Form schwach brackigen Wassers und von eutrophen Seen. Alkaliphil.

\section{Achnanthes kolbei Hustedt}

Seltene Art, die früher in Norddeutschland im Süsswasser gefunden worden ist. Die Beobachtungen an der Küste des Finnischen Meerbusens weisen (Abb. 7) darauf hin, dass die Art eine Form süssen und schwach brackigen Wassers ist.

\section{Achnanthes kriegeri Krasske}

Form süssen, kalten Wassers, die von Krasske (1949) aus Tornio-Lappland, aus der Gegend des Sees Kilpisjärvi, dargestellt worden ist.

\section{Achnantbes kryopbila Petersen}

Eine in Finnland verhältnismässig häufige Art in Seen und Flüssen sowie an feuchten Moosen. (Abb. 7.)

\section{Achnanthes lacunarum Hustedt}

Seltene kleine Form wahrscheinlich süssen Wassers, die in Finnland nur in Kemi-Lappland (Mölder) festgestellt worden ist.

\section{Achnanthes lanceolata (Breb.) Grunow}

Synonyme: Acbnanthes baynaldi var. elliptica-lanceolata, var. oblongo-elliptica Schaarschmidt, $A$. lanceolata fo. ventricosa Hustedt.

Häufige Art in süd- und nordfinnischen Seen, Flüssen, Bächen, Quellen und an der Küste in schwach salzhaltigem Wasser (Abb. 7). Alkaliph le Form. Kosmopolitische Verbreitung, u. a. in Japan (Okuno 1953), Südamerika (Frenguelli 1945), Indien (Gandhi 1962), Nordamerika (Patrick \& Reimer 1966), Südafrika (Cholnoky 1960).

\section{Fo. capitata O. Müller}

Synonyme: Acbnanthes haynaldi var. vulgaris Schaarschmidt. Achnanthes lanceolata var. baynaldi Cleve.

Neben der Hauptform, aber bedeutend seltener. 


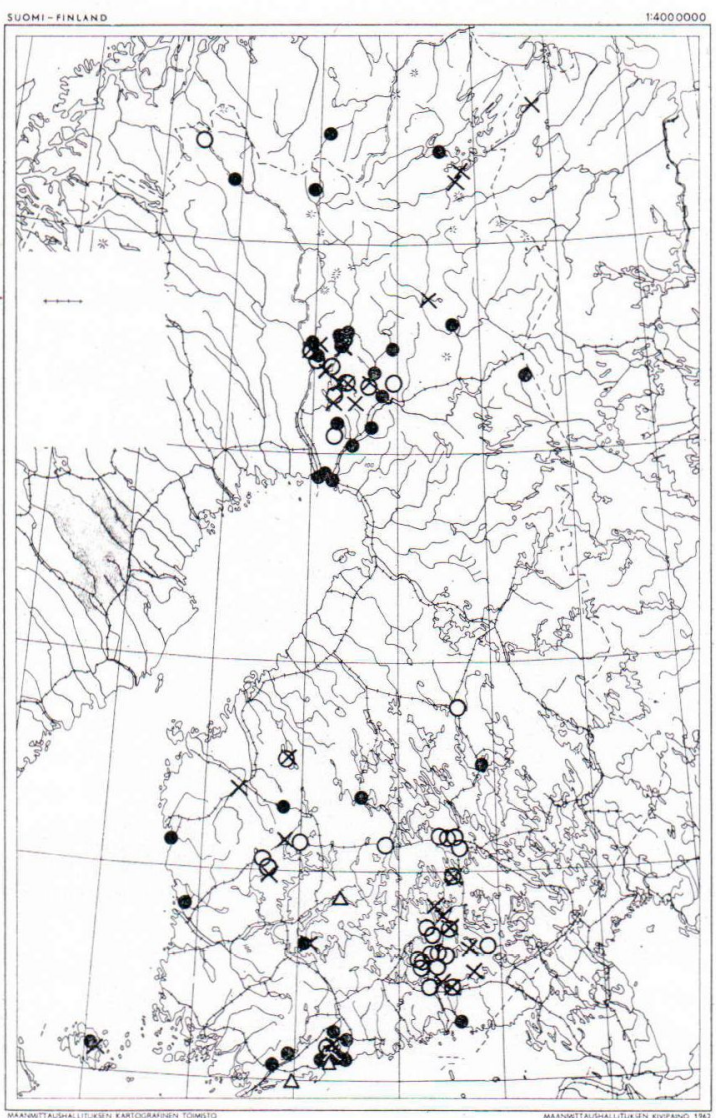

Авв. 7. Rezente Verbreitung von Achnanthes kolbei (Dreiecke), A. kryophila (Ringe), A. lanceolata (Punkte) und $A$. lapponica (Kreuze).

\section{Var. elliptica Cleve}

Häufige Variation in Nord- und Südfinnland, meistens neben der Hauptform.

\section{Var. fennica A. Cleve}

Cleve-Euler (1934) hat die Variation aus Finnisch-Lappland, dem Gebiet von Inari, sowie vom Oberlauf des Tenojoki beschrieben.

\section{Var. rostrata (Östrup) Hustedt}

Verhältnismässig häufige Variation.

\section{Achnanthes lapidosa Krasske}

Krasske (1949) hat die Art in Gebiet von

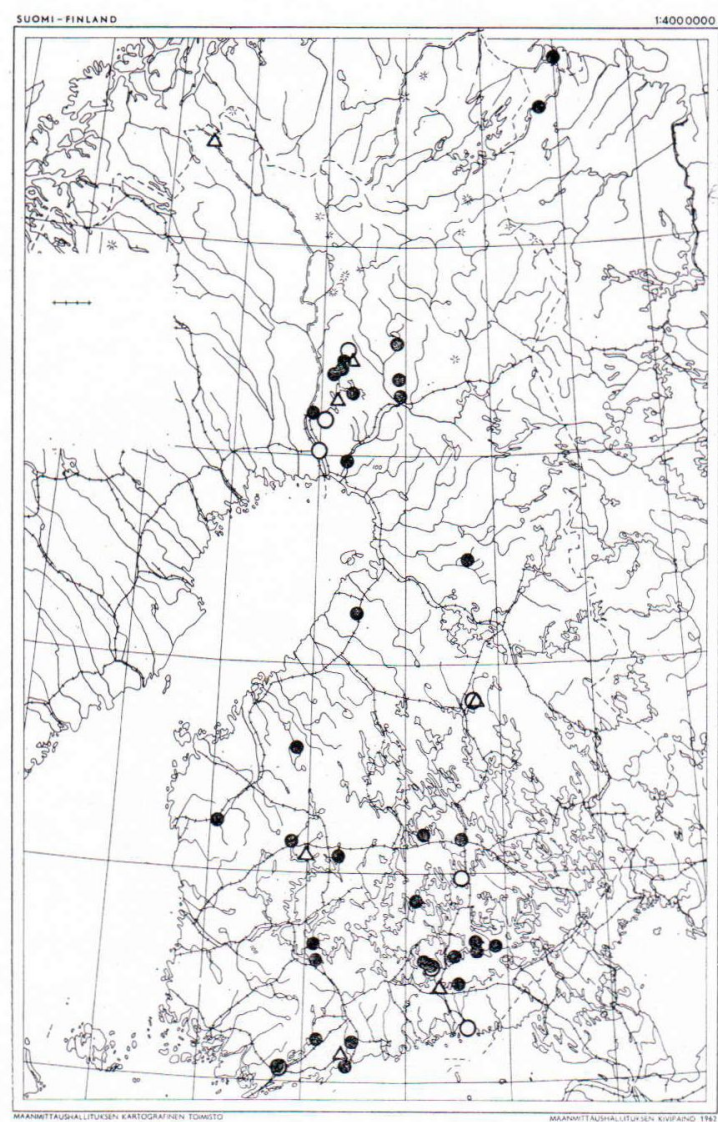

Авв. 8. Rezente Verbreitung von Achnanthes laterostrata (Dreiecke), $A$. lemmermanni (Ringe) und $A$. levanderi Punkte).

Kilpisjärvi in einem kleinen Tümpel angetroffen. Subfossil häufiger.

\section{Achnanthes lapponica Hustedt}

Synonym: Eucocconeis lapponica Hustedt.

Eine Form, die in Lappland und Südfinnland in vielen Flüssen, Seen sowie ausserdem auf feuchten Felsen und im Moos wächst (Abb. 7). Acidophile Form.

\section{Achnanthes laterostrata Hustedt}

Seltene Süsswasserform (Abb. 8).

Subfossil in Süsswassersedimenten festgestellte Art. 


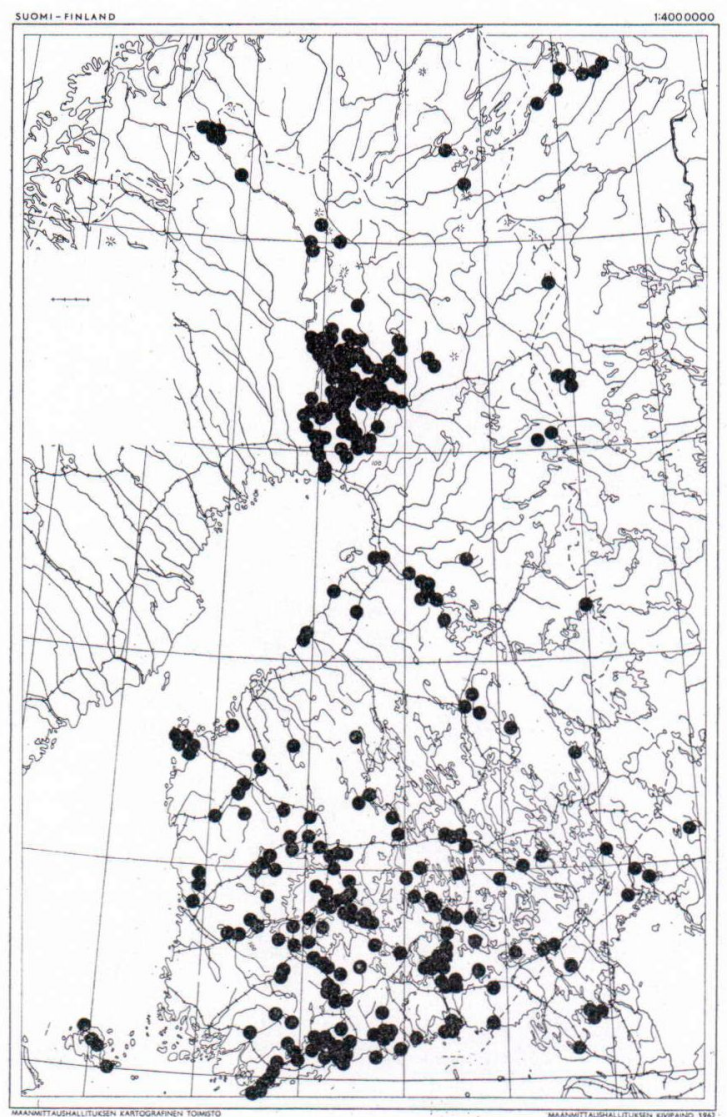

Авв. 9. Rezente Verbreitung von Acbnanthes linearis.

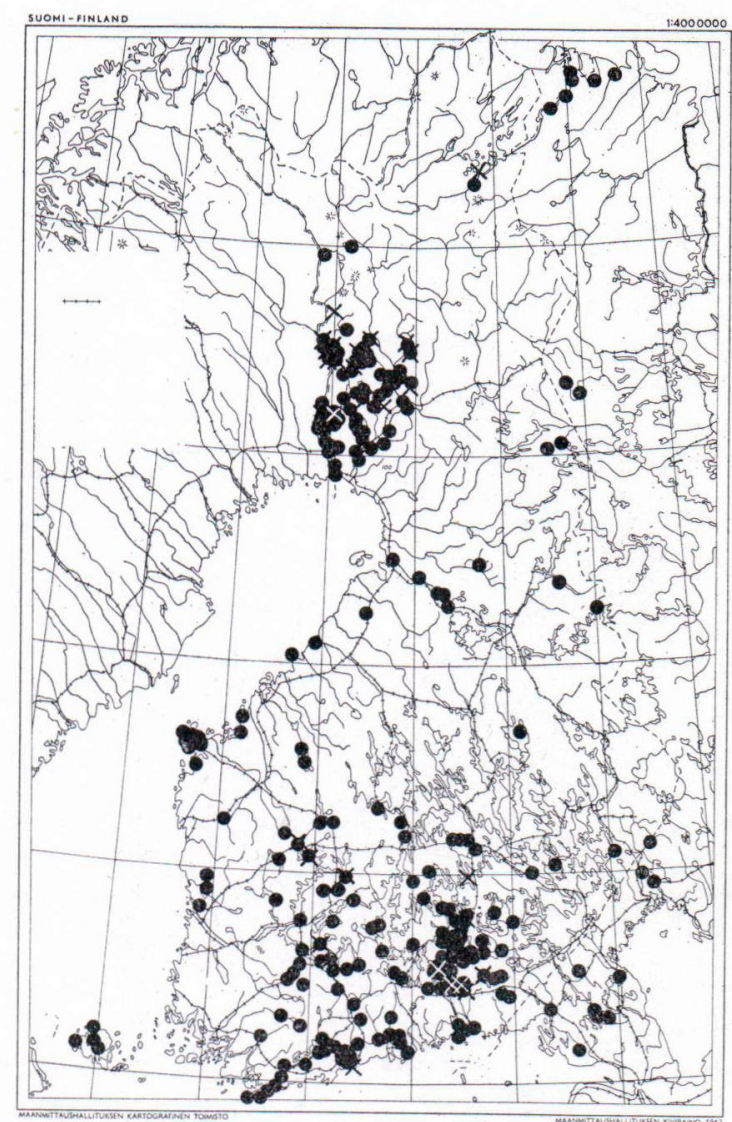

Авв. 10. Rezente Verbreitung von Achnanthes lutheri (Kreuze) und $A$. microcepbala (Punkte).

\section{Var. belvetica Hustedt}

Neben der Hauptform selten.

Achnanthes linearis (W. Smith) Grunow und. var. pusilla Grunow

Die häufigsten Acbnanthes-Formen Nordeuropas, gemein in ganz Finland in Ufergebiet vieler Binnengewässer, in Bächen, Quellen, Moosen, aber auch in schwach salzhaltigem Küstenwasser angetroffen (Abb. 9). Bei diesen allgemeinen Süsswasserformen mögen die Beobachtungen in marinen Gebiet teilweise Allochton sein, aber wegen ihrer reichlichen Vorkommen im Planton in der Pojo-Bucht (Mölder 1958) sind die Art und Variation oligohalobe Formen. 


\section{Achnanthes longipes Agradh}

Kosmopilitische Form von Salz- und Brackwasser (Filho et al. 1971), die vor Vaasa, Tammisaari und Helsinki festgestellt worden ist. Subfossil vorwiegend in Litorina- und Postlitorinasedimenten.

Fo. lata Peragallo

In Grundsedimenten des Bottnischen Meerbusens subfossil nur einmal wahrgenommen.

\section{Achnanthes lutheri Hustedt}

Kleine aus Finnland dargestellte rel. seltene Süsswasserform. (Abb. 10.)

\section{Achnanthes marginulata Grunow}

Seltene nordisch-alpine Art, deren Vorkommen u. a. an Moos und feuchten Felsen in Lappland haufiger ist als entsprechend in Südfinnland.

\section{Var. sublaevis (Hustedt) Cleve-Euler}

\section{Synonym: Achnanthes sublaevis Hustedt.}

Nordische Form, von Krasske (1949) auf den Fjelden Saanatunturi und Jehkastunturi gefunden.

\section{Achnanthes maxima A. Cleve}

Der A. flexella sehr ähnliche Form des Nordens und gebirgigen Gebiets, aber wegen ihrer beträchtlichen Grösse und der vollständigeren Symmetrie ihres Valvarhabitus als eigene Art unterschieden. U. a. in einem See südlich des Inarijärvi aufgefunden (Cleve-Euler 1953).

\section{Achnanthes microcephala Kützing}

Synonym: Microneis microcephala Cleve.

In ganz Finnland eine gemeine Süsswasserform. (Abb. 10.)

\section{Achnanthes minutissima Kützing}

Synonym: Microneis minutissima Cleve.

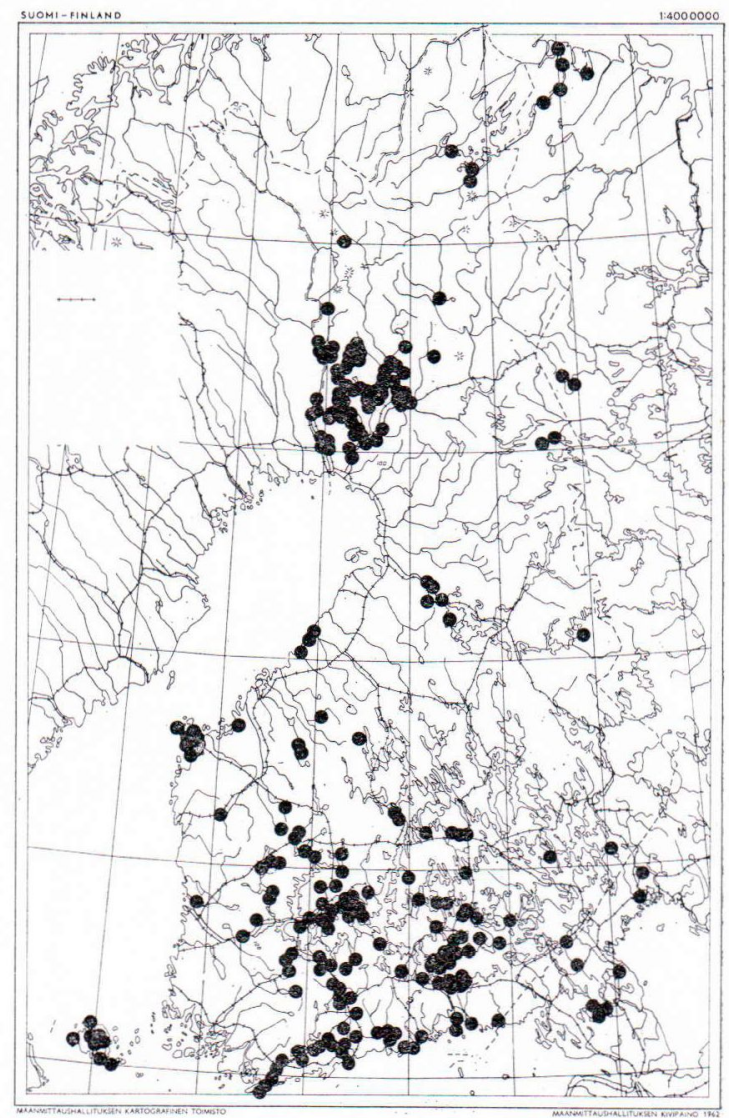

Авв. 11. Rezente Verbreitung von Achnanthes minutissima.

Sehr häufige Süsswasserform. Die Beobachtungen im Küstengebiet dürften teilweise autochthone Vorkommen sein. (Abb. 11.)

\section{Var. cryptocephala Grunow}

Eine neben der Hauptart oft auftretende Form.

\section{Achnanthes montana Krasske}

Nur von zwei Stellen in Nordfinnland: aus dem Oulujoki und dem östlich Aavasaksa fliessenden Tengeliönjoki eingebrachte seltene alpine Süsswasserform. 


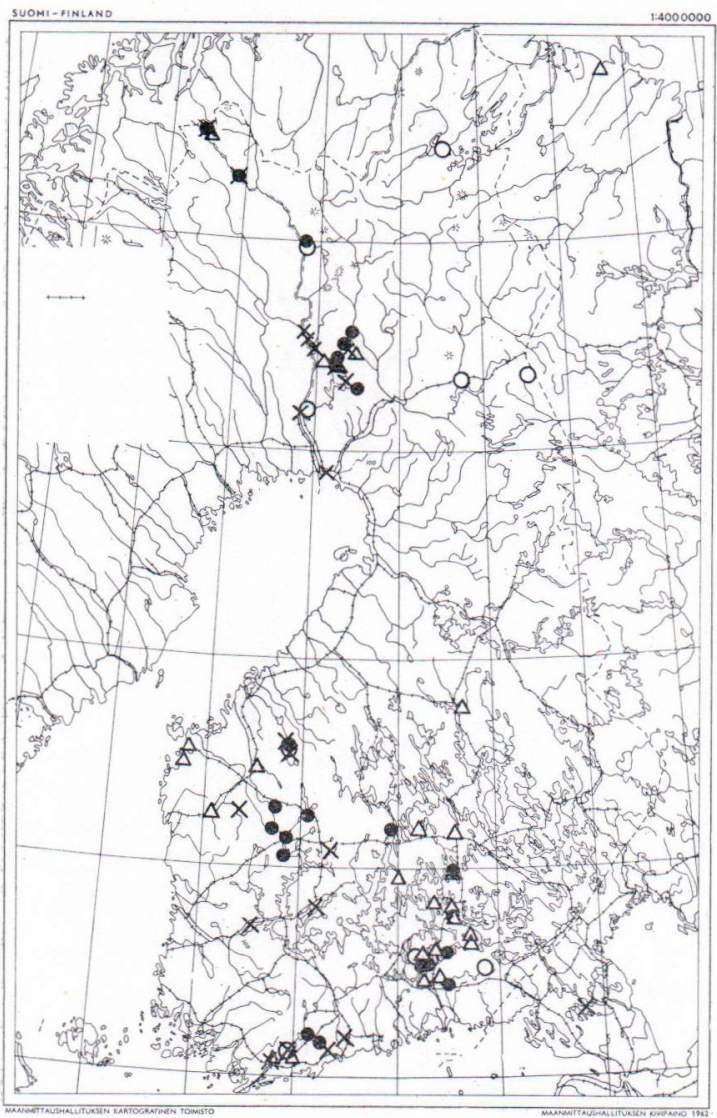

Авв. 12. Rezente Verbreitung von Acbnanthes nodosa (Punkte), A. obliqua (Ringe), A. peragalli (Kreuze) und A. saxonica (Dreiecke).

Achnanthes nodosa A. Cleve

Nordisch-alpine Süsswasserform. Mehrere Beobachtungen von Lappland bis nach Südfinnland. (Abb. 12.)

\section{Achnanthes obliqua (Greg.) Hustedt}

Synonym: Stauroneis obliqua Gregory.

Nordeuropäische Süsswasserform. Beobachtungen von Lappland bis Südfinnland. (Abb. 12.)

\section{Achnanthes peragalli Brun \& Hèribaud}

Eine von Südfinnland bis Lappland rel. häufige Form in Seen, Flüssen, Moosen. (Abb. 12.)
Achnanthes peterseni Hustedt

Krasske (1949) hat die Art in Lappland im Siilasjärvi im Moos angetroffen. Nordisch-alpin.

\section{Achnanthes recurvata Hustedt}

Eine $\operatorname{der} A$. marginulata ähnliche Form, aber an den Enden mehr zugespitzt. Nach Krasske eine in Iappland häufige Diatomeenform. Nach Hustedt ist das in Norddeutschland im Garrensee angetroffene Vorkommen ein Glazialrelikt. Meriläinen (1967) hat die Art ausserdem aus dem meromiktischen Valkiajärvi dargestellt.

\section{Achnanthes rbynchocephala A. Cleve}

Der $A$. peragalli ähnelnde Form. Von jener in erster Linie dadurch unterschieden, das die Pseudoraphe schmal und linear ist. Wahrscheinlich ist die Form im übrigen allgemein der $A$. peragalli angeschlossen.

\section{Achnanthes rupestris Krasske}

Kleine, nordisch-alpine Form, die von Krasske auf den Fjelden Saanatunturi und Jehkastunturi verhältnismässig allgemein im Moosrasen und an Felsen festgestellt worden ist.

\section{Achnanthes saxonica Krasske}

Kleine Süsswasserform, die auch an Steinen und in nassen Moosrasen wächst. In Finnland relativ häufig. (Abb. 12.)

\section{Acbnanthes septata A. Cleve}

Arktisch-marine Form, von Brander (1941) u. a. im interglazialen Ton von Rouhiala nachgewiesen.

\section{Achnanibes subsalsa Petersen}

Ursprünglich auf Island an Fadenalgen gefundene Süsswasserform. In Finnland an 9 Stellen wahrgenommen. (Abb. 13.) 


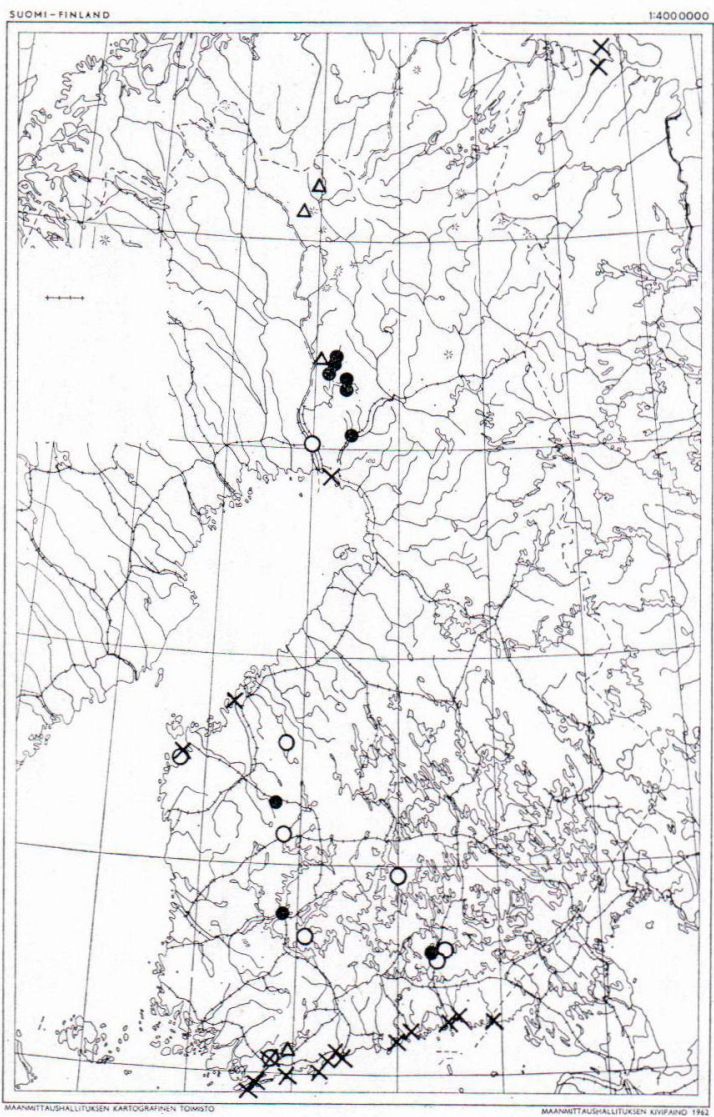

Aвв. 13. Rezente Verbreitung von Acbnantbes subsalsa (Ringe), $A$, taeniata (Kreuze), $A$. trinodis (Dreiecke) und A. östrupi (Punkte).

\section{Achnanthes subsaisoides Hustedt}

Eine nur in Eigentlich-Finnland gefundene seltene Achnanthes-Art.

\section{Acbnanthes sucblandti Hustedt}

Seltene nordisch-alpine Süsswasserform. U. a. im Gebiet von Kilpisjärvi (Krasske) sowie im Luirojoki gefunden. Subfossil häufiger festgestellt.

\section{Achnanthes taeniata Grunow}

Planktonform in Brack- und Salzwasser von der Nordküste Mitteleuropas bis zum Nördlichen Eismeer. Kaltwasserform. An der Küste des Finnischen Meerbusens häufig, am Bottnischen Meerbusen bis in die Höhe von Uusikaarlepyy häufiger (Abb. 13).

\section{Achnanthes trinodis (W. Smith) Grunow}

Süsswasserform, die u. a. im Lohjanjärvi (Hustedt) und in Westlappland festgestellt worden ist (Abb. 13).

\section{Acbnanthes östrupi (A. Cl.) Hustedt}

Synonym: Acbnanthes lanieolata var. östrupi A. Cleve. Süsswasserform, rezent selten, hauptsächlich in Nordfinnland (Abb. 13).

\section{ERGÄNZENDE SCHRIFTEN}

Carter, John, R. (1970) Observations of some British forms of Achnanthes saxonica Krasske. - Microscopy: J. Quekett micr. Club (1970), 31, 313-6.

Cholnoky, B. J. (1960) Beiträge zur Kenntnis der Ökologie der Diatomeen in dem Swartkops-Bache nahe Port Elizabeth (Südost-Kaapland). - Hydrobiologia, Acta Hydrobiologica Hydrographica et Protistologia. Vol. XVI, N. 3.

Cleve-Euler (1935) Subfossila diatomaceer från Åland. Mem. Soc. Fauna \& Fl. Fennica 10. 1933-34, Helsinki.

Donner, J. \& R. Gardemeister (1971) Redeposited Eemian marine clay in Somero, South-Western Finland. Appendix, R. Tynni: The diatoms in the Somero clay. Bull. Geol. Soc. Finland 43, 73-88.
Filho, H. M., I. M. V. Moreira, A. A. Pajares, I. I. M. Trippia (1971) Diatomaceas do Porto Salaverry. Boletin da Universidade Federal do Parana. Botanica 26, Maio 1971.

Florin, MAJ- Britt (1970) Late-Glacial diatoms of Kirschner Marsh Southeastern Minnesota. Beihefte zur Nova Hedwigia, Hf. 31, J. Cramer 1970.

Frenguelli, JoAquin (1945) El Platense y sus Diatomeas. Revista del Museo de la Plata, Tomo II, Geologia, N. 17.

GandHI, H. P. (1962) The Diatom-Flora of the Bombay and Salsette Islands II. Nova Hedwigia III, 4, J. Cramer 1962.

- (1964) The Diatonflora of Chandola and Kankaria Lakes. Nova Hedwigia VIII, 3/4, S. 349-402. 
Hessle, Chr. \& S. Wallin (1934) Undersökningar över plankton och dess växlingar i Östersjön under åren 1925-1927. Sv. Hydrogr. biol. Komm. skr. N. S. Biologi, Bd. 1,5. Stockholm.

Hustedt, FR. (1933) Die Kieselalgen Deutschlands, Österreichs und der Schweiz. 2. Teil, Lif. 3. Rabenhors: Kryptogamen-Flora, 7. Leipzig.

- (1950) Die Diatomeenflora norddeutscher Seen mit besonderer Berücksichtigung des holsteinischen Seegebiets 5-7. Arch. Hydrobiol. 43: 329-458.

- (1962) Die Kieselalgen Deutschlands, Osterreichs und der Schweiz. 3. Teil, Lif. 2. Rabenhorst: KryptogamenFlora, 7. Leipzig.

Iwaki, Sumie (1968) Diatoms from the Isuzu River in Ise. Bull. Japonese Soc. Phycology, Vol. XVI, N. 1, April 1968.
JurILJ, A. (1954) Flora i Vegetacija Diatomeja Ohridskog Jezera. Jugoslavenska Akademija Znanosti i Umjetnosti Zagreb 1954.

Okuno, H. (1953) Electron microscopical study of fine structures of diatom frustules X. Bot. Magazine, Vol. LXVI, N. 775-776.

Zabelina M. M., Kiselev I. A., Proškina-Lavrenko, A. I., Šešukova V. S., 1951.

ЗАБЕЛИНА М. М., КИСЕЛЕВ И. А., ПРОШКИНА-ЛАВРЕНКОИ А.И., ШЕШУКОВА В.С. 1951: Днатомовые водоросли. Определитель пресноводых водорослей СССР, вып. 4. Изд.Сов.наука. - 619 рр. Москва.

Manuskript eingegangen am 24. Februar 1972. 
TAFEL I
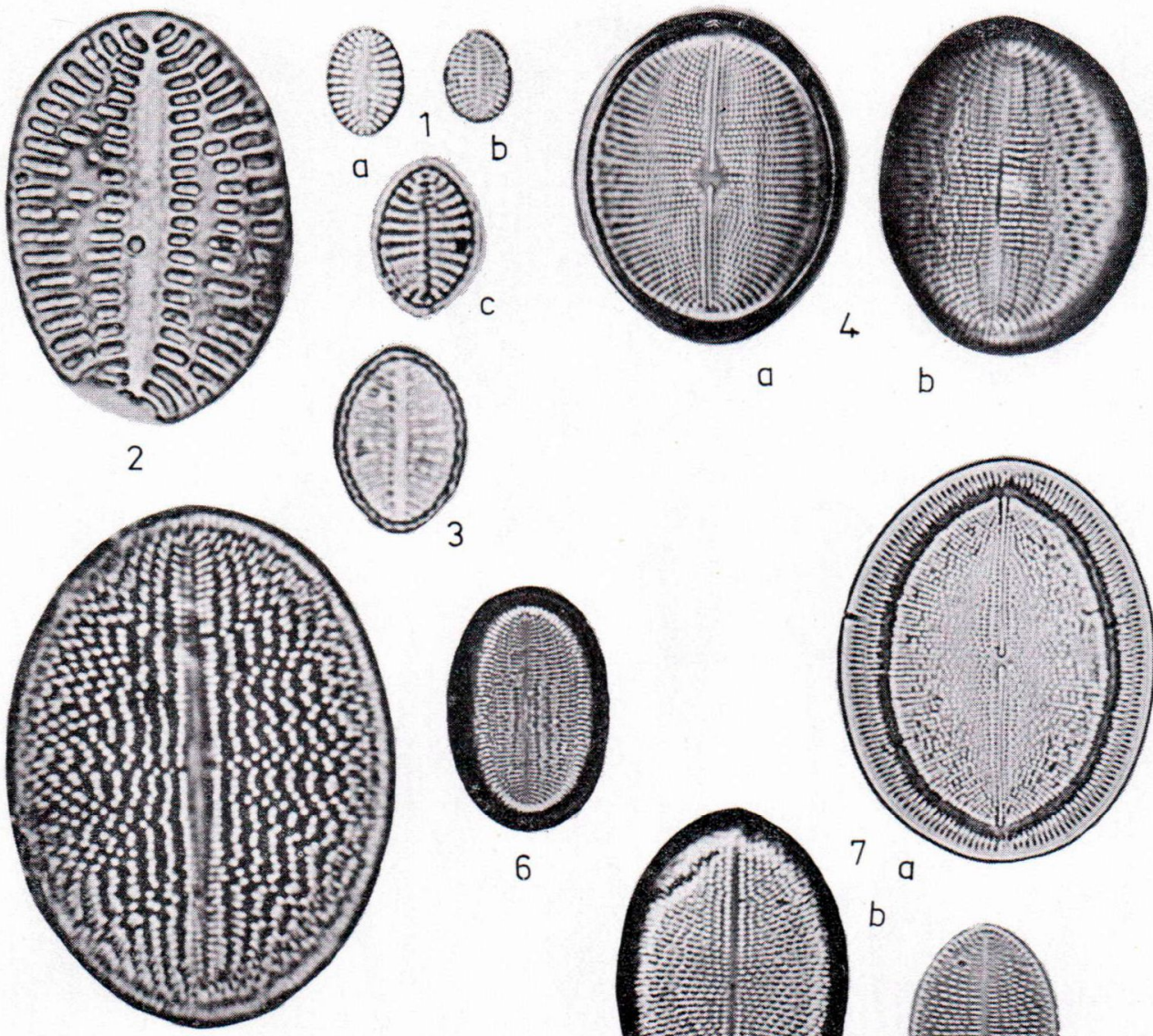

b

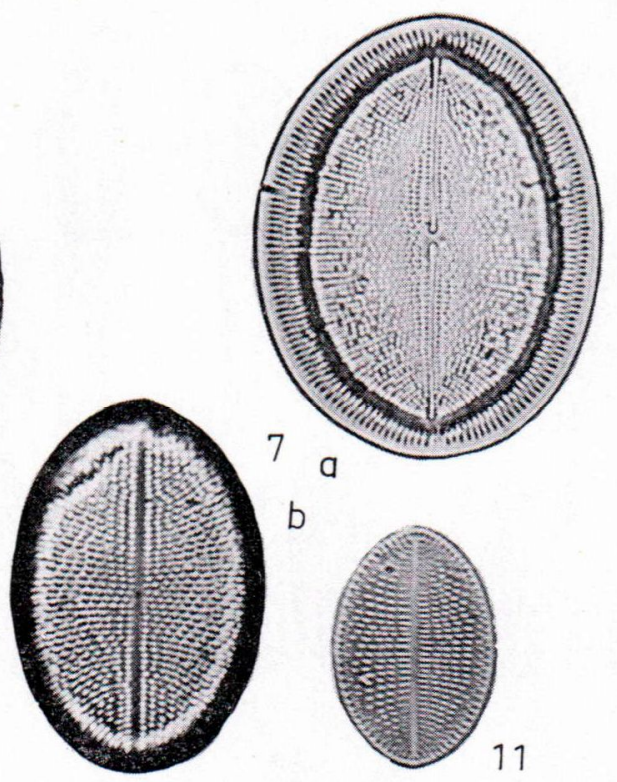

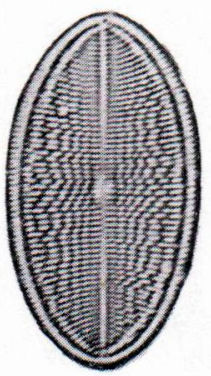

8

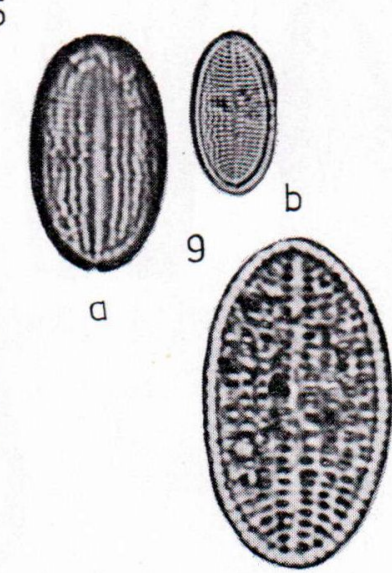

10
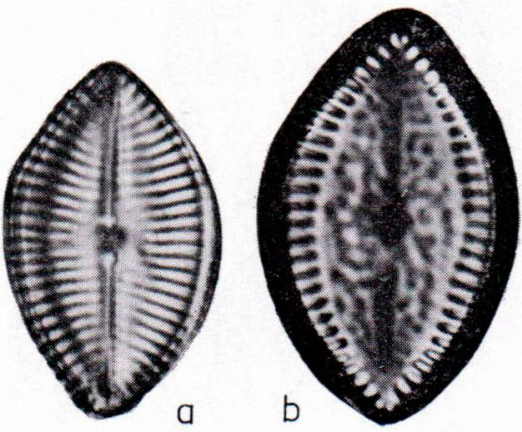

12

1. a-c Cocconeis diminuta, 2. C. disculus, 3. C. d. var. minor, 4. a-b, 5-6. C. pediculus, 7. a-b, 8. C. placentula, 9. a-b C. p. var euglypta, 10. C. p. var intermedia, 11. C. p. var. linearis, 12. a-b C. quarnerensis. 
TAFEL II
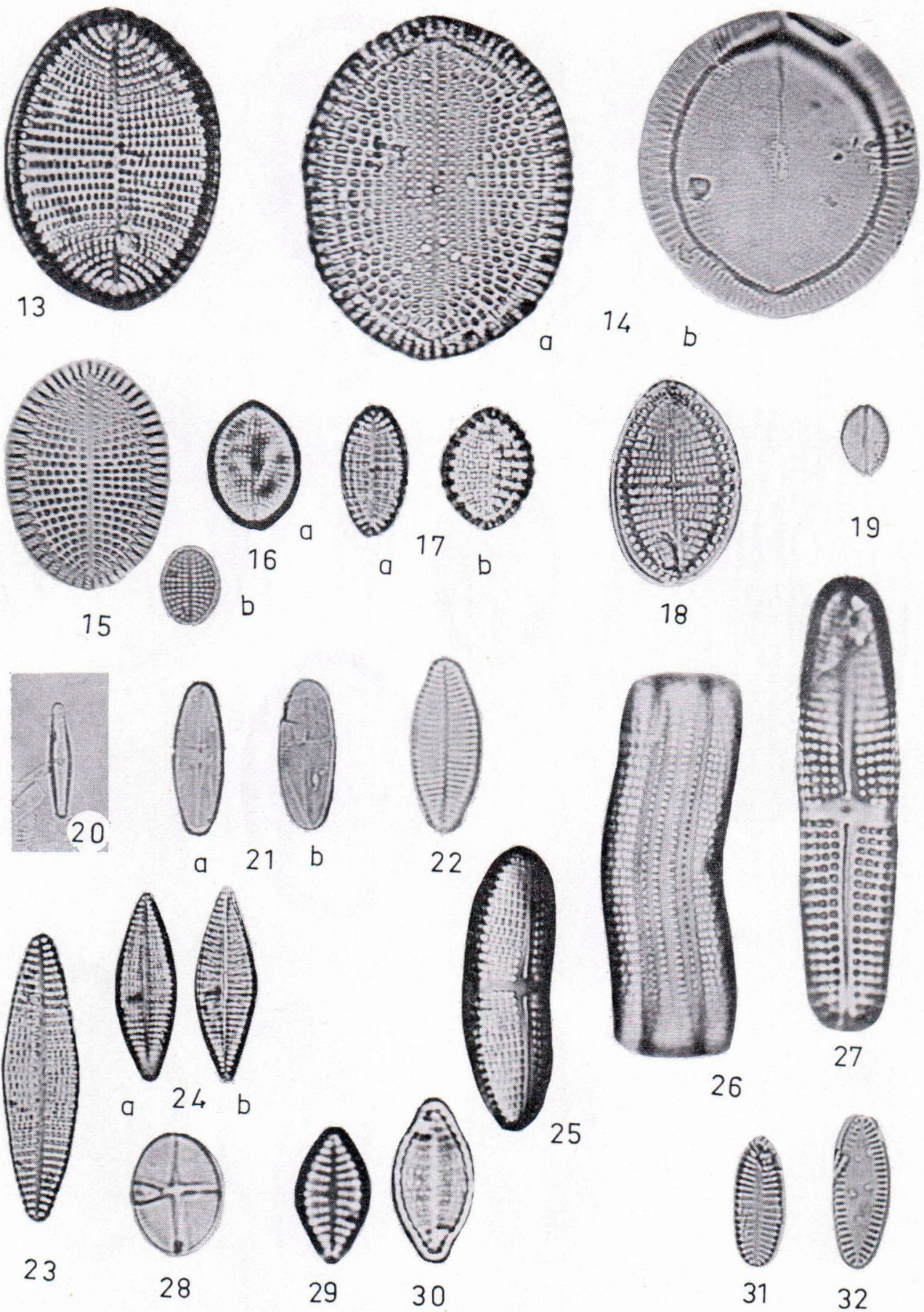

13, 14. a-b, 15. Cocconeis scutellum, 16. a-b C. s. var. minutissima, 17. a-b C. s. var. parva, 18. C. s. var. stauroneiformis, 19. C. thumensis, 20. Achnanthes affinis, 21. a-b A. austriaca var. belvetica, 22. A. biasolettiana, 23, 24. a-b A. bottnica, 25-26. A. brevipes, 27. A. b. var. intermedia, 28. A. calcar, 29. A. clevei 30. A. c. var. rostrata ,31. A. conspicua, 32. A. c. var brevistriata. 
TALEF III

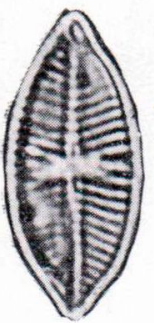

33

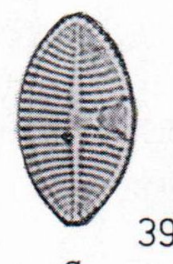

a

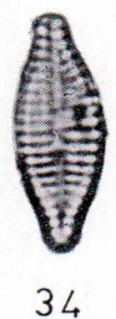

34
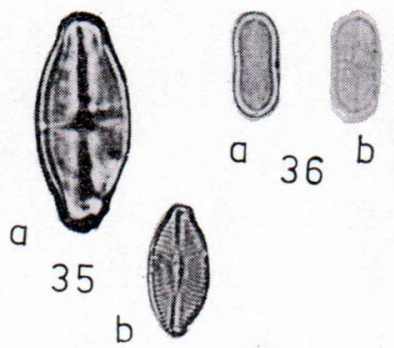

(1)
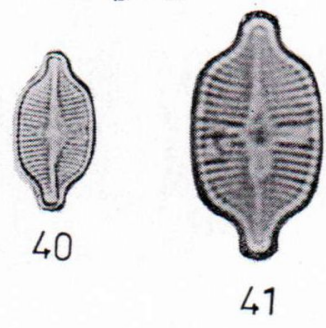

b
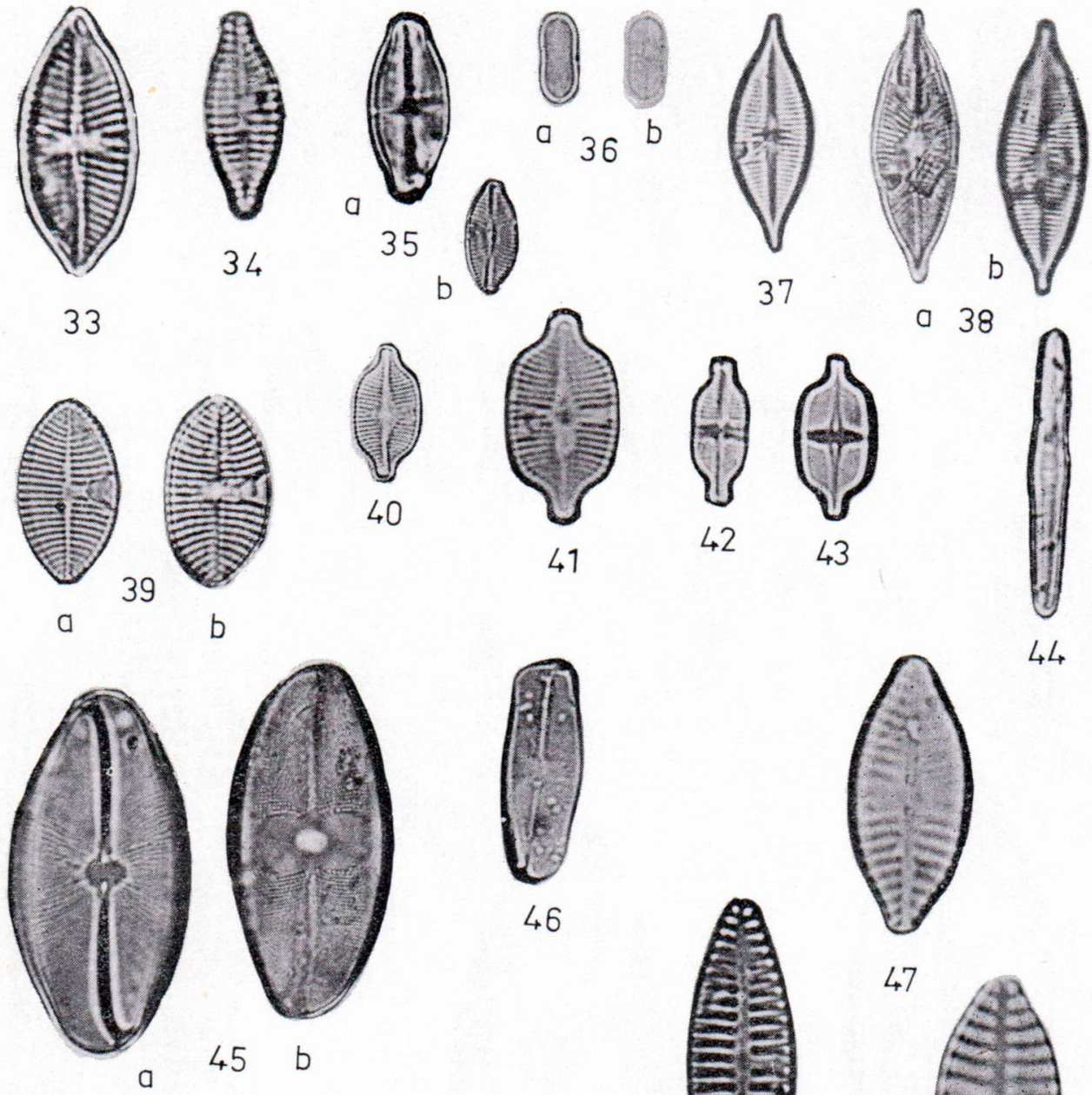

व 38

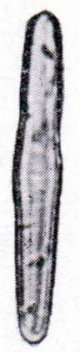

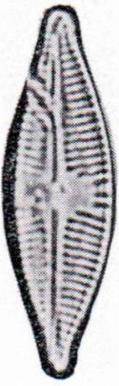

व

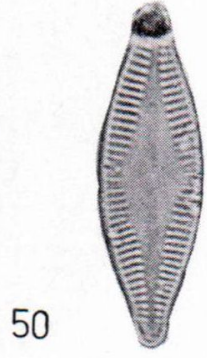

b

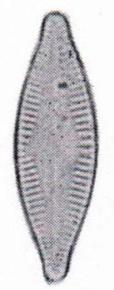

51

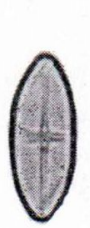

52
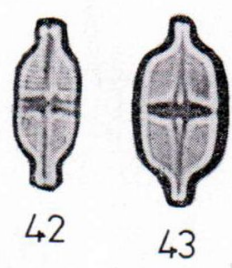

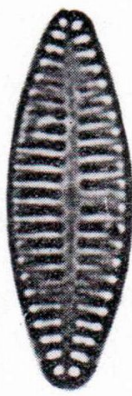

48

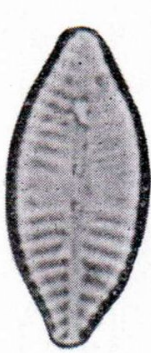

47

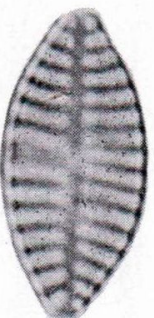

49

33-34. Acbnanthes delicatula, 35. a-b A. depressa, 36. a-b A. didyma, 37. A. dispar, 38. a-b A. d. var. angulata, 39. a-b A. elliptica, 40-41. A. exigua, 42. A. e. var. constricta, 43. A. e. var. beterovalvata, 44. A. exilis, 45. a-b A. flexella, 46. A. f. var. alpestris, 47-49. A. bauckiana, 50. a-b, 51. A. bolsti, 52. A. bungarica, 53. A. kolbei, 54. a-c A. kryophila. 
TAFEL IV
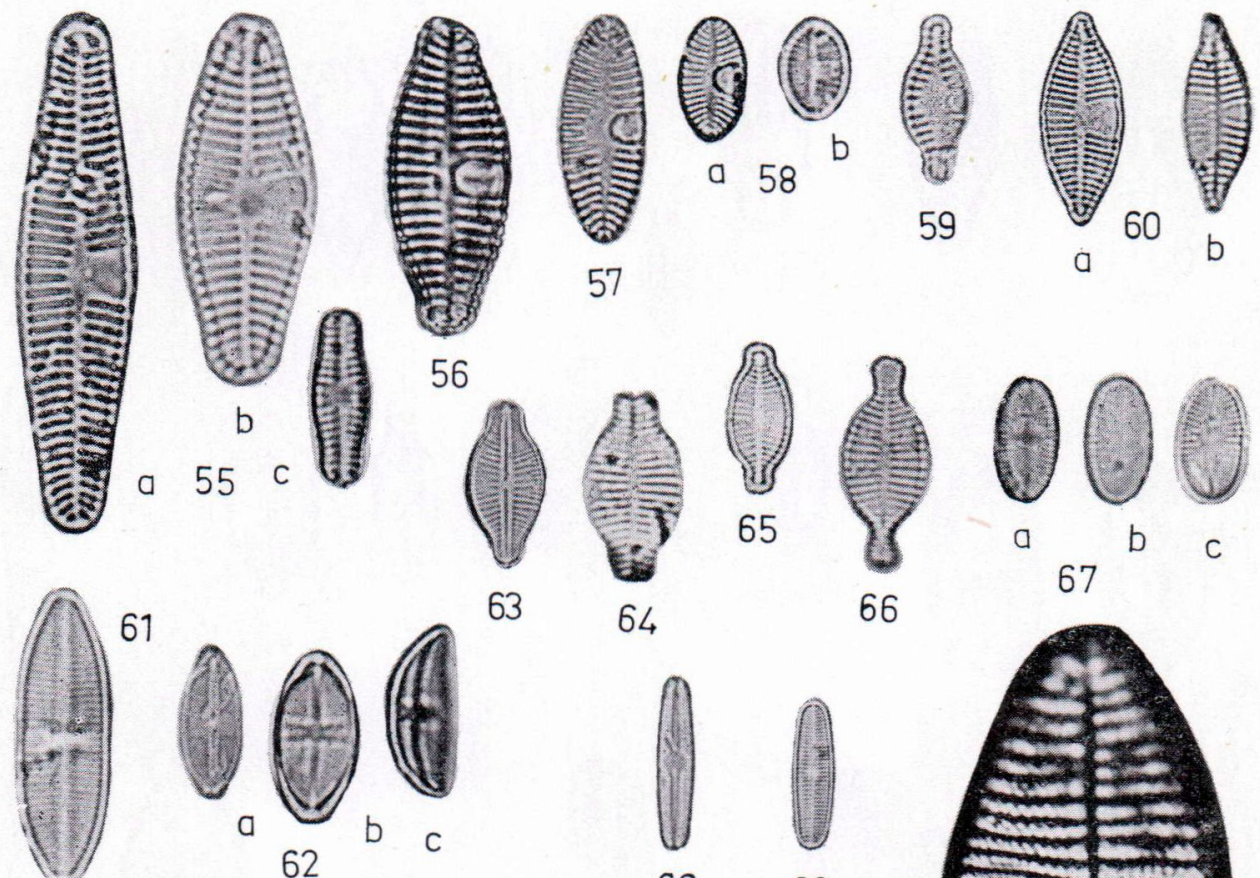

63

64

66 67

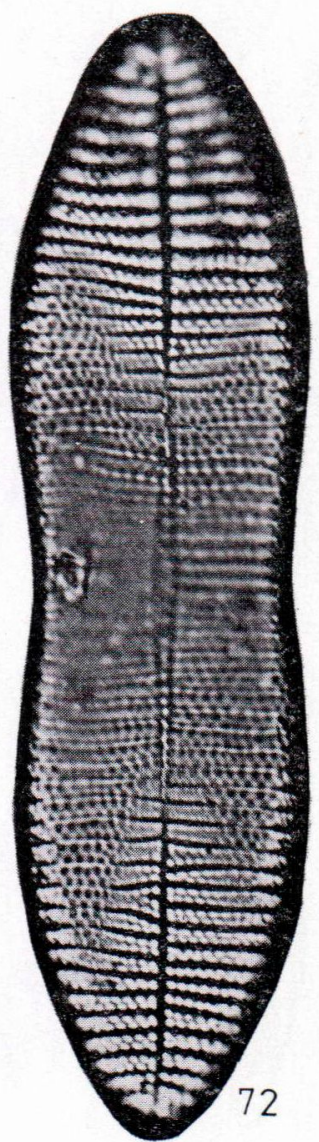

55. a-c, 56. Achnanthes lanceolata, 57, 58, a-b A. l. var. elliptica, 59, 60. a-b A. l. var. rostrata, 61. A. lapidosa, 62. a-c A. lapponica, 63-65. A. laterostrata, 66. A. lemmermanni, 67. a-b A. levanderi, 67. c A. $l$. var. belvetica, 68. A. linearis, 69. A. l. var. pusilla, 70, 72. A. longipes, 71. Übergangsform nach A. brevipes. 

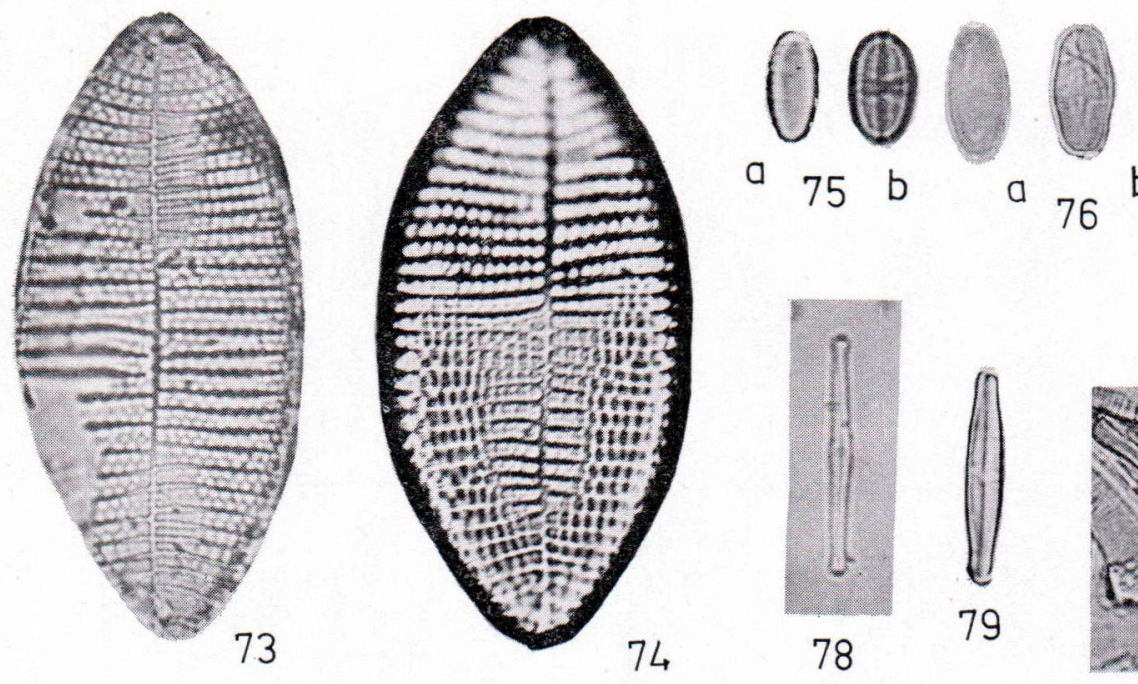

TAFEL V
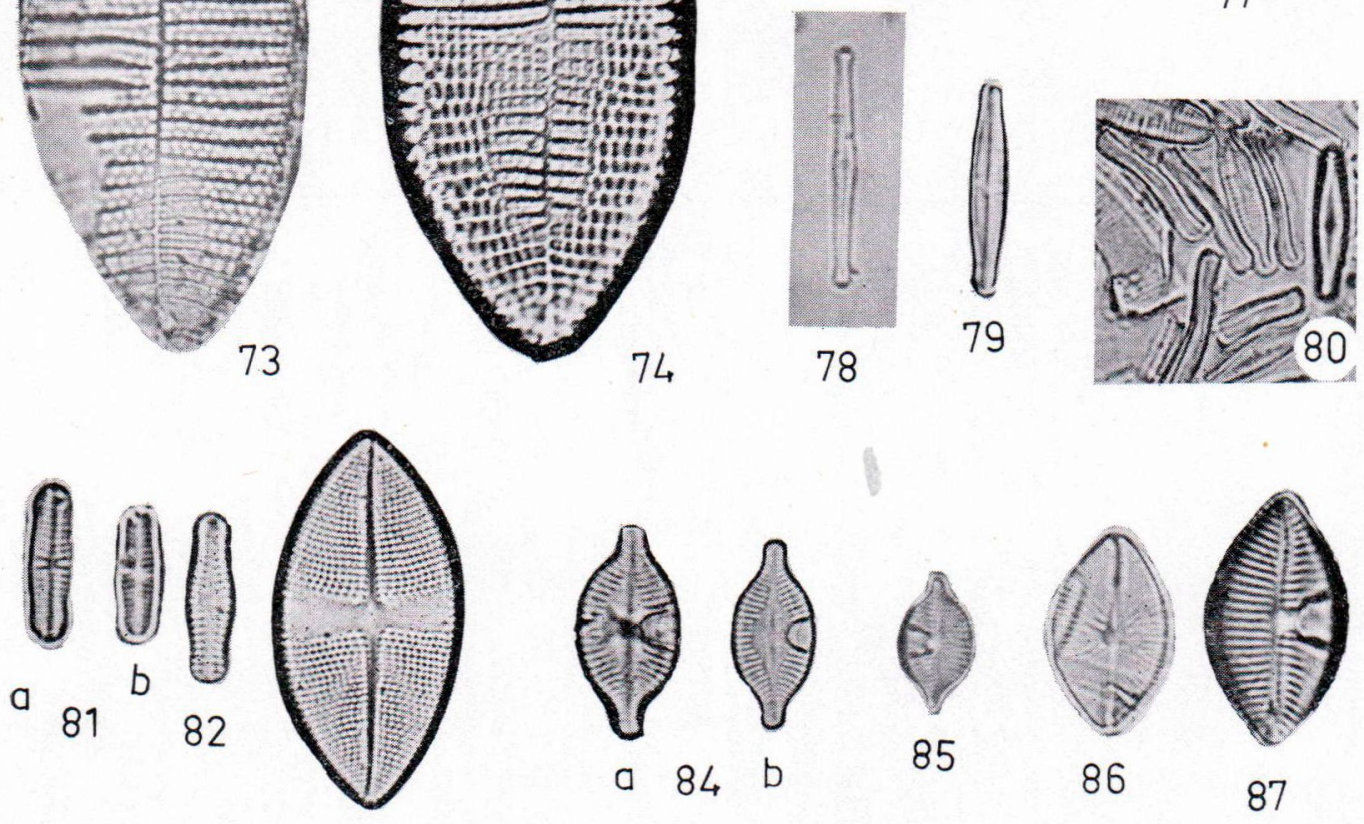

83
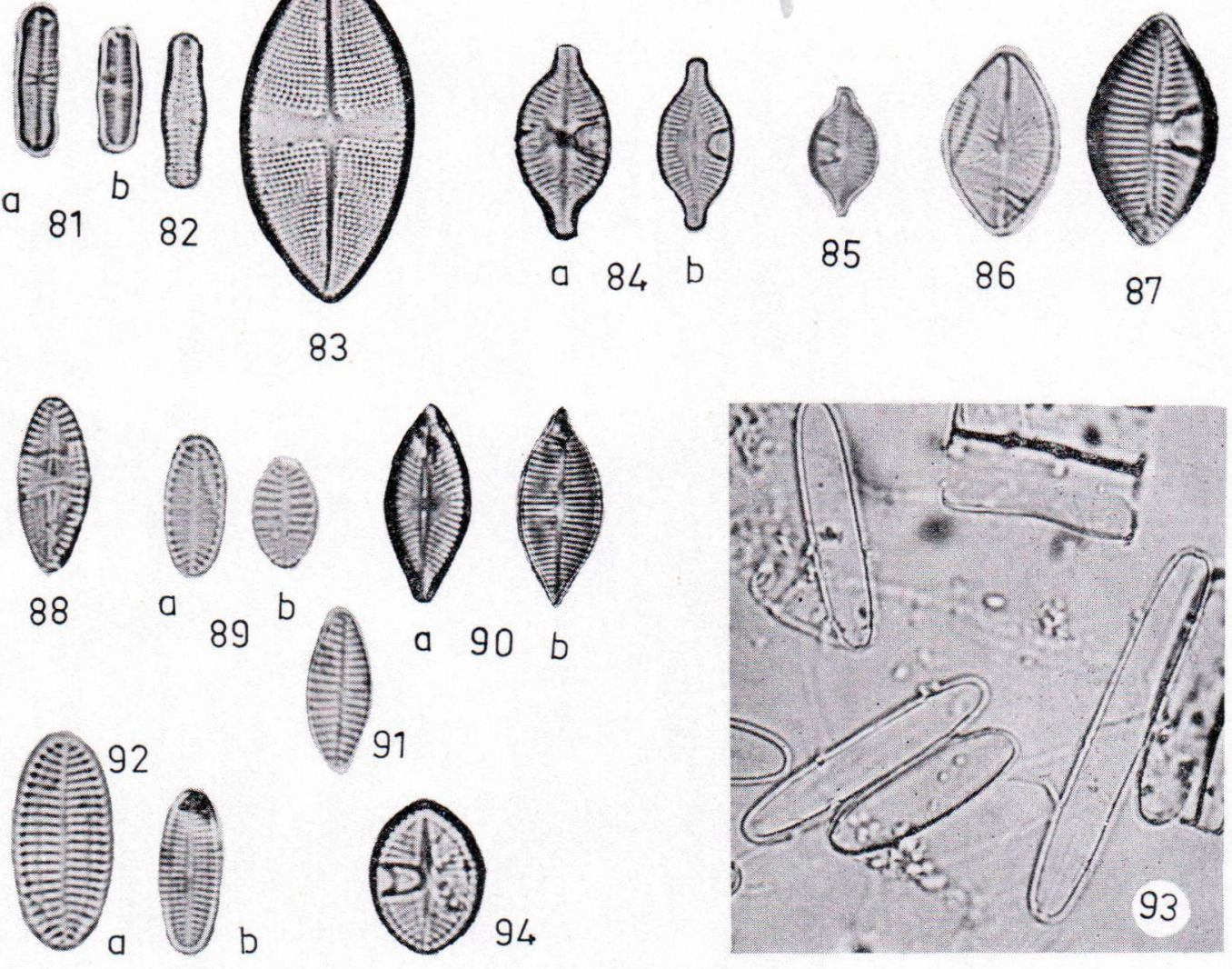

73-74. Achnanthes longipes fo. lata, 75. a-b A. lutheri, 76. a-c A. marginulata, 77. A. m. var. sublaevis, 78. A. microcephala, 79-80. A. minutissima var. cryptocephala, 81. a-b, 82. A. nodosa, 83. A. obliqua, 84. a-b A. peragalli, 85-87. A. rbynchocephala, 88. A. rupestris, 89. a-b. A. saxonica, 90. a-b $A$. subsalsa, 91. A. subsalsoides, 92. a-b A. sucblandti, 93. A. taeniata, 94. A. östrupi. Photo R. Tynni und E. Halme. (etwa 1000 -fache Vergr., mit Ausnahme von Fig. 25, 26400 -fache, 1. c, 2, 36. a-b, 41, 54. a-c, 62. a-c, 67. a-c 1500 -fache). 